\title{
THE SINGLE-VALUED EXTENSION PROPERTY AND SPECTRAL MANIFOLDS
}

\author{
SHAN LI SUN
}

(Communicated by Palle E. T. Jorgensen)

\begin{abstract}
We discuss the relation between the single-valued extension property (that is, Dunford's property (A)) and spectral manifolds $X_{T}(F)$ of a bounded linear operator. In particular, we prove that Dunford's property $(C)$ implies the property (A). We also prove that if $T \in B(X)$ has the property $\left(\beta^{*}\right)$ introduced by Fong, then $X_{T^{*}}^{*}(F)=X_{T}(\mathbb{C} \backslash F)^{\perp}$ for every closed set $F$ in the complex plane $\mathbb{C}$.
\end{abstract}

In the spectral decomposition theory of bounded linear operators the singlevalued extension property is an elementary and important property. All spectral, decomposable, and hyponormal operators have this property, but there are some ordinary operators which do not have this property, for example, the adjoint of the unilateral shift on a Hilbert space. For an operator $T \in B(X)$ without the single-valued extension property we may also define the spectral manifold $X_{T}(F)$. In this case the properties of $X_{T}(F)$ are different than ones of operators with the single-valued extension property. In the first part of this paper we discuss some dependent relations between the single-valued extension property and spectral manifolds $X_{T}(F)$. In particular, we prove that Dunford's property (C) implies his property (A), that is, the single-valued extension property, and so we strengthen an essential and important result in the theory of spectral operators due to Dunford and Schwartz [2]. In the second part of the paper we prove that if for every open covering $\left\{G_{1}, \ldots, G_{n}\right\}$ of $\sigma(T)$,

$$
X=X_{T}\left(\bar{G}_{1}\right)+\cdots+X_{T}\left(\bar{G}_{n}\right),
$$

then $X_{T^{*}}^{*}(F)=X_{T}(\mathbb{C} \backslash F)^{\perp}$ for each closed set $F$ in the complex plane $\mathbb{C}$. So we generalize and deepen a main result in the duality theory due to Frunză [4].

In this paper $\mathbb{C}$ denotes the complex plane, $X$ the complex Banach space, and $B(X)$ the Banach algebra of all bounded linear operators on $X$. If $T \in$ $B(X)$ and $F$ is a closed set in $\mathbb{C}$, we define

$$
\begin{aligned}
& X_{T}(F)=\{x \in X ; \text { there exists an analytic } X \text {-valued function } \\
& \qquad f: \mathbb{C} \backslash F \rightarrow X \text { such that }(\lambda-T) f(\lambda)=x, \lambda \in \mathbb{C} \backslash F\} .
\end{aligned}
$$

$X_{T}(F)$ is said to be the spectral manifold of $T$. If $G$ is an open set in $\mathbb{C}$, we

Received by the editors June 3, 1991.

1991 Mathematics Subject Classification. Primary 47B40. 
define

$$
X_{T}(G)=\bigcup\left\{X_{T}(F) ; F \subseteq G \text { and } F \text { is closed }\right\},
$$

where " $U$ " denotes the linear span. If $T$ has the single-valued extension property, then the above definition is identical with Dunford and Schwartz's original definition

$$
X_{T}(F)=\left\{x \in X ; \sigma_{T}(x) \subseteq F\right\},
$$

where $\sigma_{T}(x)$ is the local spectrum of $T$ at $x$.

It is well known that if $T \in B(X)$ has the single-valued extension property, then the following propositions are true:

(1) For arbitrary closed sets $F_{1}$ and $F_{2}$ in $\mathbb{C}$,

$$
X_{T}\left(F_{1}\right) \cap X_{T}\left(F_{2}\right)=X_{T}\left(F_{1} \cap F_{2}\right) \text {. }
$$

(2) If $X_{T}(F)$ is closed for a closed set $F$ in $\mathbb{C}$, then

$$
\sigma\left(T \mid X_{T}(F)\right) \subseteq \sigma(T) \cap F
$$

(3) If $X_{T}(F)$ is closed for a closed set $F$ in $\mathbb{C}$, then $X_{T}(F)$ is an analytic invariant subspace of $T$, that is, $X_{T}(F)$ is an invariant subspace of $T$ and $f(\lambda) \in X_{T}(F)$ for an arbitrary analytic $X$-valued function $f: G \rightarrow X$ on some open set $G$ in $\mathbb{C}$ satisfying $(\lambda-T) f(\lambda) \in X_{T}(F), \lambda \in G$.

(4) If $X_{T}(F)$ is closed for a closed set $F$ in $\mathbb{C}$, then $X_{T}(F)$ is the spectral maximal space of $T$, that is, $X_{T}(F)$ is an invariant subspace of $T$ and for an arbitrary invariant subspace $Y$ of $T$,

$$
\sigma(T \mid Y) \subseteq \sigma\left(T \mid X_{T}(F)\right) \text { implies } Y \subseteq X_{T}(F)
$$

If $T$ does not have the single-valued extension property, then these propositions are false.

Example 1. Let $H$ be a separable complex Hilbert space, $\left\{e_{n}\right\}_{n=-\infty}^{+\infty}$ the orthonormal basis of $H$, and $T \in B(H)$ a bilateral weighted shift on $H$ :

$$
T e_{n}=w_{n} e_{n+1}, \quad n=0, \pm 1, \pm 2, \ldots,
$$

where $\left\{w_{n}\right\}_{n=-\infty}^{+\infty}$ is a monotone decreasing sequence of positive numbers and $w_{n} \nearrow 1$ as $n \rightarrow-\infty, w_{n} \searrow \frac{1}{2}$ as $n \rightarrow+\infty$. It follows from [5] that $T^{*}$ is a hyponormal operator and

$$
\sigma(T)=\left\{\lambda \in \mathbb{C} ; \quad \frac{1}{2} \leq|\lambda| \leq 1\right\} .
$$

It follows from [6] that $T$ does not have the single-valued extension property. We write

$$
\begin{array}{cc}
F_{1}=\{\lambda \in \mathbb{C} ;|\lambda|=1\}, & F_{2}=\left\{\lambda \in \mathbb{C} ;|\lambda|=\frac{1}{2}\right\}, \\
H_{k}^{+}=\bigvee_{n=k}^{+\infty}\left\{e_{n}\right\}, & H_{k}^{-}=\bigvee_{n=-\infty}^{k}\left\{e_{n}\right\},
\end{array}
$$

where " $\bigvee$ " denotes the closed linear span.

Proposition 1. For arbitrary integer $k$,

$$
H_{k}^{-} \subseteq X_{T}\left(F_{1}\right), \quad H_{k}^{+} \subseteq X_{T}\left(F_{2}\right) .
$$


Proof. Since $H_{k}^{+}$is an invariant subspace of $T, T \mid H_{k}^{+}$is a unilateral weighted shift with the weight sequence $\left\{w_{n}\right\}_{n=k}^{+\infty}$, and $w_{n} \searrow \frac{1}{2}$ as $n \rightarrow+\infty$, it follows from [5] that

$$
\sigma\left(T \mid H_{k}^{+}\right)=\left\{\lambda \in \mathbb{C} ;|\lambda| \leq \frac{1}{2}\right\}
$$

For arbitrary $x \in H_{k}^{+},\left(\lambda-T \mid H_{k}^{+}\right)^{-1} x$ is an analytic $X$-valued function on $\left\{\lambda \in \mathbb{C} ;|\lambda|>\frac{1}{2}\right\}$ and

$$
(\lambda-T)\left(\lambda-T \mid H_{k}^{+}\right)^{-1} x=x, \quad|\lambda|>\frac{1}{2} .
$$

Because $\sigma(T)=\left\{\lambda \in \mathbb{C} ; \frac{1}{2} \leq|\lambda| \leq 1\right\}$, we can define

$$
f_{x}(\lambda)= \begin{cases}(\lambda-T)^{-1} x, & |\lambda|<\frac{1}{2}, \\ \left(\lambda-T \mid H_{k}^{+}\right)^{-1} x, & |\lambda|>\frac{1}{2}\end{cases}
$$

then $f_{x}(\lambda)$ is an analytic $X$-valued function on $\mathbb{C} \backslash F_{2}$ and

$$
(\lambda-T) f_{x}(\lambda)=x, \quad \lambda \in \mathbb{C} \backslash F_{2} .
$$

So $x \in X_{T}\left(F_{2}\right)$, that is, $H_{k}^{+} \subseteq X_{T}\left(F_{2}\right)$.

For the first inclusion we note that $T$ is invertible and

$$
T^{-1} e_{n}=w_{n-1}^{-1} e_{n-1}, \quad n=0, \pm 1, \pm 2, \ldots,
$$

so for arbitrary integer $k, H_{k}^{-}$is an invariant subspace of $T^{-1}$. Analogously we can prove $H_{k}^{-} \subseteq X_{T^{-1}}\left(F_{1}\right)$. It follows from the definition of the spectral manifold that $X_{T^{-1}}\left(F_{1}\right)=X_{T}\left(F_{1}\right)$, since $F_{1}$ is the unit circle. Hence $H_{k}^{-} \subseteq$ $X_{T}\left(F_{1}\right)$, so the proof is complete.

It follows from Proposition 1 that $X_{T}\left(F_{1}\right) \cap X_{T}\left(F_{2}\right) \neq\{0\}$. But $X_{T}\left(F_{1} \cap F_{2}\right)=$ $\{0\}$, since $F_{1} \cap F_{2}=\varnothing$. Therefore statement (1) is false.

Choosing $F=F_{1} \cup F_{2}$, it follows from Proposition 1 that

$$
X_{T}(F)=X_{T}\left(F_{1}\right)+X_{T}\left(F_{2}\right)=H .
$$

Hence $\sigma\left(T \mid X_{T}(F)\right)=\sigma(T) \nsubseteq F$, that is, statement (2) is false.

For statement $(3)$ we choose $F=\varnothing$. Then $X_{T}(F)=\{0\}$ is not an analytic invariant subspace of $T$.

Example 2. Let $T_{1}$ be the bilateral weighted shift in Example $1, T_{2}=\frac{2}{3} U$, where $U$ is the bilateral shift (nonweighted) on $H$, and $X=H \oplus H, T=$ $T_{1} \oplus T_{2}, F=F_{1} \cup F_{2}$, where $F_{1}, F_{2}$ are the closed sets in Example 1. Then $T \in$ $B(X)$ does not have the single-valued extension property, $X_{T}(F)=H \oplus\{0\}$, and $\sigma\left(T \mid X_{T}(F)\right)=\sigma\left(T_{1}\right)$. Note that $\sigma(T)=\sigma\left(T_{1}\right) \cup \sigma\left(T_{2}\right) \subseteq \sigma\left(T_{1}\right)$, that is, $\sigma(T) \subseteq \sigma\left(T \mid X_{T}(F)\right)$, but $X=H \oplus H \supsetneq X_{T}(F)$. Hence $X_{T}(F)$ is not the spectral maximal space of $T$.

Regardless of whether a bounded linear operator $T$ has the single-valued extension property, the following propositions are true.

Proposition 2. For an arbitrary closed set $F$ in $\mathbb{C}, X_{T}(F)$ is a hyperinvariant manifold of $T$. In particular, if $X_{T}(F)$ is closed, then it is a hyperinvariant subspace of $T$.

Proof. Suppose $x \in X_{T}(F)$. Then there exists an analytic $X$-valued function $f: \mathbb{C} \backslash F \rightarrow X$ such that

$$
(\lambda-T) f(\lambda)=x, \quad \lambda \in \mathbb{C} \backslash F
$$


So $S f(\lambda)$ is also an analytic $X$-valued function on $\mathbb{C} \backslash F$ for each $S \in B(X)$ commuting with $T$, and

$$
(\lambda-T) S f(\lambda)=S(\lambda-T) f(\lambda)=S x, \quad \lambda \in \mathbb{C} \backslash F .
$$

Hence $S x \in X_{T}(F)$, so the proof is complete.

Proposition 3. If $X_{T}(F)$ is closed for a closed set $F$ in $\mathbb{C}$, then

$$
\sigma\left(T \mid X_{T}(F)\right) \subseteq \sigma(T) \cap \widehat{F},
$$

where $\widehat{F}$ is the union of $F$ and all bounded components in $\mathbb{C} \backslash F$.

Proof. Suppose $\lambda_{0} \notin \widehat{F}$. To show $\lambda_{0} \notin \sigma\left(T \mid X_{T}(F)\right)$, it is sufficient to prove that $\lambda_{0}-T \mid X_{T}(F)$ is bijective. According to the definition of $X_{T}(F)$, it is surjective. If $x \in X_{T}(F)$ satisfies $\left(\lambda_{0}-T\right) x=0$, then there exists an analytic $X$-valued function $f: \mathbb{C} \backslash F \rightarrow X$ such that

$$
(\lambda-T) f(\lambda)=x, \quad \lambda \in \mathbb{C} \backslash F \text {. }
$$

Let

$$
g(\lambda)=\frac{1}{\lambda-\lambda_{0}} x, \quad \lambda \neq \lambda_{0} .
$$

Then $g(\lambda)$ is an analytic $X$-valued function on $\left\{\lambda \in \mathbb{C} ; \lambda \neq \lambda_{0}\right\}$ and

$$
(\lambda-T) g(\lambda)=x, \quad \lambda \neq \lambda_{0} .
$$

We define

$$
G(\lambda)= \begin{cases}f(\lambda), & \lambda \in \mathbb{C} \backslash \widehat{F}, \\ g(\lambda), & \lambda \neq \lambda_{0} .\end{cases}
$$

Note that each component of $\mathbb{C} \backslash \widehat{F}$ is connected with $\rho(T)$, the resolvent set of $T$, and both $f(\lambda)$ and $g(\lambda)$ are analytic continuations of $R(\lambda, T) x, \lambda \in \rho(T)$; hence

$$
f(\lambda)=g(\lambda), \quad \lambda \in(\mathbb{C} \backslash \widehat{F}) \backslash\left\{\lambda_{0}\right\} .
$$

It shows that $G(\lambda)$ is well defined. Because $G(\lambda)$ is analytic on the whole complex plane and

$$
(\lambda-T) G(\lambda)=x, \quad \lambda \in \mathbb{C},
$$

$x \in X_{T}(\varnothing)=\{0\}$, that is, $x=0$. The proof is complete.

Theorem 1. Suppose that $F_{1}$ and $F_{2}$ are two disjoint closed sets in $\mathbb{C}$. Then

$$
X_{T}\left(F_{1} \cup F_{2}\right)=X_{T}\left(F_{1}\right)+X_{T}\left(F_{2}\right) \text {. }
$$

Proof. We may assume without loss of generality that $F_{1} \cup F_{2} \subseteq \sigma(T)$. If $x \in$ $X_{T}\left(F_{1} \cup F_{2}\right)$, then there exists an analytic $X$-valued function $f: \bar{C} \backslash\left(F_{1} \cup F_{2}\right) \rightarrow X$ such that

$$
(\lambda-T) f(\lambda)=x, \quad \lambda \in \mathbb{C} \backslash\left(F_{1} \cup F_{2}\right) .
$$

For two arbitrary disjoint smoothed closed curves $C_{1}$ and $C_{2}$ in the interior of the curve $C=\{\lambda \in \mathbb{C} ;|\lambda|=\|T\|+1\}$ such that $C_{j}$ surrounds $F_{j}, j=1,2$, respectively, we have

$$
\begin{aligned}
x & =\frac{1}{2 \pi i} \int_{C}(\lambda-T)^{-1} x d \lambda=\frac{1}{2 \pi i} \int_{C} f(\lambda) d \lambda=\frac{1}{2 \pi i} \int_{C_{1} \cup C_{2}} f(\lambda) d \lambda \\
& =\frac{1}{2 \pi i} \int_{C_{1}} f(\lambda) d \lambda+\frac{1}{2 \pi i} \int_{C_{2}} f(\lambda) d \lambda .
\end{aligned}
$$


Let $x_{j}=(1 / 2 \pi i) \int_{C_{j}} f(\lambda) d \lambda$. Because $f(\lambda)$ is analytic, the $x_{j}$ are independent of the selection of the curves $C_{j}, j=1,2$. It is sufficient to prove $x_{j} \in$ $X_{T}\left(F_{j}\right), j=1,2$.

For arbitrary $\mu \in \mathbb{C} \backslash F_{1}$ there exists a smoothed closed curve $J$ surrounding $F_{1}$ such that both $\mu$ and $F_{2}$ are outside $J$. Let

$$
g(\mu)=-\frac{1}{2 \pi i} \int_{J} \frac{f(\lambda)}{\lambda-\mu} d \lambda
$$

Then $g(\mu)$ is independent of the selection of the curve $J$. It is easily seen that the function $g$ is analytic on $\mathbb{C} \backslash F_{1}$ and

$$
\begin{aligned}
(\mu-T) g(\mu) & =\frac{1}{2 \pi i} \int_{J} \frac{(T-\mu) f(\lambda)}{\lambda-\mu} d \lambda \\
& =\frac{1}{2 \pi i} \int_{J} \frac{(T-\lambda) f(\lambda)}{\lambda-\mu} d \lambda+\frac{1}{2 \pi i} \int_{J} f(\lambda) d \lambda \\
& =\frac{1}{2 \pi i} \int_{J} \frac{-x}{\lambda-\mu} d \lambda+x_{1}=x_{1}, \quad \mu \in \mathbb{C} \backslash F_{1} .
\end{aligned}
$$

So $x_{1} \in X_{T}\left(F_{1}\right)$. The proof that $x_{2} \in X_{T}\left(F_{2}\right)$ is analogous. The proof is complete.

In the case that $T$ has the single-valued extension property and $X_{T}\left(F_{1} \cup F_{2}\right)$ is closed, this result is well known. Now we have proved the result without any assumption.

Theorem 2. Suppose that $T \in B(X)$ and $X_{T}(F)$ is closed for every closed set $F$ in $\mathbb{C}$. Then $T$ has the single-valued extension property.

Proof. Let $f: G \rightarrow X$ be an analytic $X$-valued function on some open set $G$ in $\mathbb{C}$ such that

$$
(\lambda-T) f(\lambda)=0, \quad \lambda \in G .
$$

We may assume without loss of generality that $G$ is connected. Choose an open disc $U$ in $G$ such that $\bar{U} \subseteq G$; then $\widehat{\bar{U}}=\bar{U}$. According to the supposition of the theorem, $X_{T}(\bar{U})$ is closed, it follows from Proposition 3 that

$$
\sigma\left(T \mid X_{T}(\bar{U})\right) \subseteq \bar{U}
$$

For arbitrary $\lambda_{0} \in U$ let $x_{0}(\lambda)=f\left(\lambda_{0}\right) /\left(\lambda-\lambda_{0}\right), \lambda \neq \lambda_{0}$. Then $x_{0}(\lambda)$ is analytic on $\mathbb{C} \backslash\left\{\lambda_{0}\right\}$ and

$$
(\lambda-T) x_{0}(\lambda)=f\left(\lambda_{0}\right), \quad \lambda \neq \lambda_{0} .
$$

Hence $f\left(\lambda_{0}\right) \in X_{T}\left(\left\{\lambda_{0}\right\}\right) \subseteq X_{T}(\bar{U})$. Because $G$ is connected and $X_{T}(\bar{U})$ is closed, it follows by means of analytic continuation that

$$
f(\lambda) \in X_{T}(\bar{U}), \quad \lambda \in G .
$$

Hence we have

$$
\left(\lambda-T \mid X_{T}(\bar{U})\right) f(\lambda)=0, \quad \lambda \in G .
$$

If $\lambda \in G \backslash \bar{U}$, then $\lambda \notin \sigma\left(T \mid X_{T}(\bar{U})\right)$; thus $f(\lambda)=0$. It follows again by means of analytic continuation that

$$
f(\lambda)=0, \quad \lambda \in G .
$$

The proof is complete. 
From Theorem 2 we know that Dunford's property (C) implies his property (A). In the theory of spectral operators due to Dunford and Schwartz [2] there is the following essential and important result.

Theorem D-S. Let $T$ be a bounded linear operator in a weakly complete space. Then $T$ is a spectral operator if and only if $T$ has properties (A), (B), (C), and (D).

According to our Theorem 2 the property (A) in this theorem may be dropped, and this theorem can be strengthened as follows.

Theorem 3. Let $T$ be a bounded linear operator in a weakly complete space. Then $T$ is a spectral operator if and only if $T$ has properties (B), (C), and (D).

\section{2}

Frunză [4] proved that if $T \in B(X)$ is decomposable, then for every closed set $F$ in $\mathbb{C}$

$$
X_{T^{*}}^{*}(F)=X_{T}(\mathbb{C} \backslash F)^{\perp},
$$

where

$$
X_{T}(\mathbb{C} \backslash F)^{\perp}=\left\{u \in X^{*} ;\langle x, u\rangle=0, \text { for each } x \in X_{T}(\mathbb{C} \backslash F)\right\} .
$$

In the following we will prove this result under a weaker condition. First we introduce the following notion due to Fong: $T \in B(X)$ is said to have the property $\left(\beta^{*}\right)$ if for every open covering $\left\{G_{1}, \ldots, G_{n}\right\}$ of $\sigma(T)$,

$$
X=X_{T}\left(\bar{G}_{1}\right)+\cdots+X_{T}\left(\bar{G}_{n}\right) .
$$

Here $X_{T}\left(\bar{G}_{k}\right), k=1, \ldots, n$, are not necessarily closed. If, in addition, all $X_{T}\left(\bar{G}_{k}\right), k=1, \ldots, n$, are closed, then $T$ is decomposable.

Fong [3] proved that the property $\left(\beta^{*}\right)$ is the duality property of Bishop's property $(\beta)$, that is, $T \in B(X)$ has the property $(\beta)$ if and only if $T^{*}$, the adjoint of $T$, has the property $\left(\beta^{*}\right)$. It is easily known that all decomposable operators have properties $(\beta)$ and $\left(\beta^{*}\right)$. The operator $T$ in Example 1 has the property $\left(\beta^{*}\right)$, but it is not decomposable. In fact, because $T^{*}$ is hyponormal, $T^{*}$ has the property $(\beta)$. It follows from the result due to Fong that $T$ has the property $\left(\beta^{*}\right)$. In addition, $T$ is not decomposable, since $T$ does not have the single-valued extension property. Thus the property $\left(\beta^{*}\right)$ is weaker than decomposable. The following theorem is a nontrivial generalization of the above result due to Frunză.

Theorem 4. Let $T \in B(X)$ have the property $\left(\beta^{*}\right)$. Then for every closed set $F$ in $\mathbb{C}$

$$
X_{T^{*}}^{*}(F)=X_{T}(\mathbb{C} \backslash F)^{\perp} .
$$

Proof. First we prove $X_{T}(\mathbb{C} \backslash F)^{\perp} \subseteq X_{T^{*}}^{*}(F)$. Because $X_{T}(\mathbb{C} \backslash F)^{\perp}$ is an invariant subspace of $T^{*}$, it is sufficient to prove

$$
\sigma\left(T^{*} \mid X_{T}(\mathbb{C} \backslash F)^{\perp}\right) \subseteq F .
$$

Let $\lambda_{0} \in \mathbb{C} \backslash F$. It is easy to show that $\lambda_{0}-T^{*} \mid X_{T}(\mathbb{C} \backslash F)^{\perp}$ is injective. In fact, if $u \in X_{T}(\mathbb{C} \backslash F)^{\perp}$ such that $\left(\lambda_{0}-T^{*}\right) u=0$, then we can show $u=0$ as follows: Suppose that $\left\{G_{1}, G_{2}\right\}$ is an open covering of $\sigma(T)$ such that

$$
\lambda_{0} \notin \bar{G}_{1}, \quad \lambda_{0} \in G_{2} \subseteq \bar{G}_{2} \subseteq \mathbb{C} \backslash F .
$$


According to the property $\left(\beta^{*}\right)$ of $T$,

$$
X=X_{T}\left(\bar{G}_{1}\right)+X_{T}\left(\bar{G}_{2}\right) .
$$

Thus for each $x \in X$ there are $x_{k} \in X_{T}\left(\bar{G}_{k}\right), k=1,2$, such that

$$
x=x_{1}+x_{2} \text {. }
$$

Because $x_{1} \in X_{T}\left(\bar{G}_{1}\right)$, there exists an analytic $X$-valued function $f_{x_{1}}(\lambda)$ on $\mathbb{C} \backslash \bar{G}_{1}$ such that

$$
x_{1}=(\lambda-T) f_{x_{1}}(\lambda), \quad \lambda \in \mathbb{C} \backslash \bar{G}_{1} .
$$

Note that $x_{2} \in X_{T}\left(\bar{G}_{2}\right) \subseteq X_{T}(\mathbb{C} \backslash F)$ and $\lambda_{0} \in \mathbb{C} \backslash \bar{G}_{1}$. Then we have

$$
\begin{aligned}
\langle x, u\rangle & =\left\langle x_{1}+x_{2}, u\right\rangle=\left\langle x_{1}, u\right\rangle=\left\langle\left(\lambda_{0}-T\right) f_{x_{1}}\left(\lambda_{0}\right), u\right\rangle \\
& =\left\langle f_{x_{1}}\left(\lambda_{0}\right),\left(\lambda_{0}-T^{*}\right) u\right\rangle=0 .
\end{aligned}
$$

Hence $u=0$.

Before proving that $\lambda_{0}-T^{*} \mid X_{T}(\mathbb{C} \backslash F)^{\perp}$ is surjective we give some primary knowledge. Choose an open covering $\left\{G_{1}, G_{2}\right\}$ of $\mathbb{C}$ such that

$$
\lambda_{0} \notin \bar{G}_{1}, \quad \lambda_{0} \in G_{2} \subseteq \bar{G}_{2} \subseteq \mathbb{C} \backslash F .
$$

Choose again another open covering $\left\{D_{1}, D_{2}\right\}$ of $\mathbb{C}$ which satisfies

$$
F \subseteq D_{1} \subseteq \bar{D}_{1} \subseteq G_{1}, \quad \lambda_{0} \in D_{2} \subseteq \bar{D}_{2} \subseteq G_{2}
$$

Because $T$ has the property $\left(\beta^{*}\right)$, we have

$$
X=X_{T}\left(\bar{D}_{1}\right)+X_{T}\left(\bar{D}_{2}\right)=X_{T}\left(\bar{G}_{1}\right)+X_{T}\left(\bar{G}_{2}\right) .
$$

Since the above four manifolds $X_{T}(\cdot)$ are not necessarily closed, we need the following discussion. For an arbitrary closed set $E$ in $\mathbb{C}$, let

$X_{T}^{b}(E)=\{x \in X$; there exists a uniformly bounded analytic $X$-valued function $f_{x}$ on $\mathbb{C} \backslash E$ such that $\left.(\lambda-T) f_{x}(\lambda)=x, \lambda \in \mathbb{C} \backslash E\right\}$.

For $x \in X_{T}^{b}(E)$ we define $\|x\|_{b}^{E}=\inf \left\{\sup \left\{\left\|f_{x}(\lambda)\right\| ; \lambda \in \mathbb{C} \backslash E\right\} ; f_{x}\right.$ is a uniformly bounded analytic $X$-valued function on $\mathbb{C} \backslash E$ such that $\left.(\lambda-T) f_{x}(\lambda)=x, \lambda \in \mathbb{C} \backslash E\right\}$.

It is easy to verify that $\|\cdot\|_{b}^{E}$ is a norm on $X_{T}^{b}(E)$ and $X_{T}^{b}(E)$ is complete under this norm; hence it is a Banach space. For a fixed $\lambda_{1} \in \mathbb{C} \backslash E$, for every $x \in X_{T}^{b}(E)$,

$$
\begin{aligned}
\|x\| & =\left\|\left(\lambda_{1}-T\right) f_{x}\left(\lambda_{1}\right)\right\| \leq\left\|\lambda_{1}-T\right\|\left\|f_{x}\left(\lambda_{1}\right)\right\| \\
& \leq\left\|\lambda_{1}-T\right\| \sup \left\{\left\|f_{x}(\lambda)\right\| ; \lambda \in \mathbb{C} \backslash E\right\} .
\end{aligned}
$$

So $\|x\| \leq\left\|\lambda_{1}-T\right\|\|x\|_{b}^{E}$, that is, there is a constant $M_{E}$ such that $\|x\| \leq$ $M_{E}\|x\|_{b}^{E}$ for every $x \in X_{T}^{b}(E)$. According to the selection of $\left\{G_{1}, G_{2}\right\}$ and $\left\{D_{1}, D_{1}\right\}$, we have

$$
X_{T}\left(\bar{D}_{k}\right) \subseteq X_{T}^{b}\left(\bar{G}_{k}\right) \subseteq X_{T}\left(\bar{G}_{k}\right), \quad k=1,2 .
$$


It follows from (1) that

$$
X=X_{T}^{b}\left(\bar{G}_{1}\right)+X_{T}^{b}\left(\bar{G}_{2}\right) .
$$

We introduce a new Banach space

$$
X^{b}=X_{T}^{b}\left(\bar{G}_{1}\right) \oplus X_{T}^{b}\left(\bar{G}_{2}\right) .
$$

The norm in $X^{b}$ is defined as

$$
\|x\|_{b}=\left\|x_{1}\right\|_{b}^{1}+\left\|x_{2}\right\|_{b}^{2}
$$

for $x=x_{1} \oplus x_{2}, x_{k} \in X_{T}^{b}\left(\bar{G}_{k}\right), k=1,2$, where $\left\|x_{j}\right\|_{b}^{j}=\left\|x_{j}\right\|_{b}^{\bar{G}_{j}}, j=1,2$.

Let $J: X^{b} \rightarrow X$ be defined as

$$
J x=x_{1}+x_{2}
$$

for $x=x_{1} \oplus x_{2}, x_{k} \in X_{T}^{b}\left(\bar{G}_{k}\right), k=1,2$. Evidently, $J$ is linear and

$$
\begin{aligned}
\|J x\| & =\left\|x_{1}+x_{2}\right\| \leq\left\|x_{1}\right\|+\left\|x_{2}\right\| \\
& \leq M_{\bar{G}_{1}}\left\|x_{1}\right\|_{b}^{1}+M_{\bar{G}_{2}}\left\|x_{2}\right\|_{b}^{2} \leq M\|x\|_{b},
\end{aligned}
$$

where $M=\max \left\{M_{\bar{G}_{1}}, M_{\bar{G}_{2}}\right\}$. This shows that $J$ is a bounded linear map from $X^{b}$ to $X$. It follows from (2) that $J$ is surjective. According to the open map theorem there is a constant $M_{b}>0$ such that for every $x \in X$ there are $x_{k} \in X_{T}^{b}\left(\bar{G}_{k}\right), k=1,2$, satisfying

$$
x=x_{1} \oplus x_{2} \text { and }\left\|x_{k}\right\|_{b}^{k} \leq M_{b}\|x\|, \quad k=1,2 .
$$

Now we can prove that $\lambda_{0}-T^{*} \mid X_{T}(\mathbb{C} \backslash F)^{\perp}$ is surjective. Suppose $u \in$ $X_{T}(\mathbb{C} \backslash F)^{\perp}$. We define $v$ as follows: For every $x \in X$ there are $x_{k} \in X_{T}^{b}\left(\bar{G}_{k}\right)$, $k=1,2$, such that $x=x_{1}+x_{2}$. Because $x_{1} \in X_{T}^{b}\left(\bar{G}_{1}\right)$, there exists a uniformly bounded analytic $X$-valued function $f_{x_{1}}$ on $\mathbb{C} \backslash \bar{G}_{1}$ such that

$$
(\lambda-T) f_{x_{1}}(\lambda)=x_{1}, \quad \lambda \in \mathbb{C} \backslash \bar{G}_{1} .
$$

Note that $\lambda_{0} \in \mathbb{C} \backslash \bar{G}_{1}$. We can define

$$
\langle x, v\rangle=\left\langle f_{x_{1}}\left(\lambda_{0}\right), u\right\rangle
$$

$v$ is well defined. In fact, suppose that there again are $y_{k} \in X_{T}^{b}\left(\bar{G}_{k}\right), k=$ 1,2 , such that $x=y_{1}+y_{2}$ and another uniformly bounded analytic $X$-valued function $f_{y_{1}}$ on $\mathbb{C} \backslash \bar{G}_{1}$ such that

$$
(\lambda-T) f_{y_{1}}(\lambda)=y_{1}, \quad \lambda \in \mathbb{C} \backslash \bar{G}_{1} .
$$

(Note: it is possible that $x_{k}=y_{k}, k=1,2$, but $f_{x_{1}}$ and $f_{y_{1}}$ are different.) Then

$$
y_{1}-x_{1}=x_{2}-y_{2} \in X_{T}^{b}\left(\bar{G}_{1}\right) \cap X_{T}^{b}\left(\bar{G}_{2}\right) \subseteq X_{T}\left(\bar{G}_{1}\right) \cap X_{T}\left(\bar{G}_{2}\right) .
$$

Note that $f_{y_{1}}-f_{x_{1}}$ is an analytic $X$-valued function on $\mathbb{C} \backslash \bar{G}_{1}$ and

$$
(\lambda-T)\left(f_{y_{1}}(\lambda)-f_{x_{1}}(\lambda)\right)=y_{1}-x_{1}, \quad \lambda \in \mathbb{C} \backslash \bar{G}_{1} .
$$

Because $y_{1}-x_{1} \in X_{T}\left(\bar{G}_{2}\right)$, there exists an analytic $X$-valued function $g$ on $\mathbb{C} \backslash \bar{G}_{2}$ such that

$$
(\lambda-T) g(\lambda)=y_{1}-x_{1}, \quad \lambda \in \mathbb{C} \backslash \bar{G}_{2} .
$$


Since $\left\{G_{1}, G_{2}\right\}$ is an open covering of $\mathbb{C},\left(\mathbb{C} \backslash \bar{G}_{1}\right) \cap\left(\mathbb{C} \backslash \bar{G}_{2}\right)=\varnothing$. So we can define

$$
G(\lambda)= \begin{cases}f_{y_{1}}(\lambda)-f_{x_{1}}(\lambda), & \lambda \in \mathbb{C} \backslash \bar{G}_{1}, \\ g(\lambda), & \lambda \in \mathbb{C} \backslash \bar{G}_{2} .\end{cases}
$$

Then $G(\lambda)$ is an analytic $X$-valued function on $\left(\mathbb{C} \backslash \bar{G}_{1}\right) \cup\left(\mathbb{C} \backslash \bar{G}_{2}\right)=\mathbb{C} \backslash\left(\bar{G}_{1} \cap \bar{G}_{2}\right)$, and

$$
(\lambda-T) G(\lambda)=y_{1}-x_{1}, \quad \lambda \in \mathbb{C} \backslash\left(\bar{G}_{1} \cap \bar{G}_{2}\right) .
$$

This shows that $y_{1}-x_{1} \in X_{T}\left(\bar{G}_{1} \cap \bar{G}_{2}\right)$, so $G\left(\lambda_{0}\right)=f_{y_{1}}\left(\lambda_{0}\right)-f_{x_{1}}\left(\lambda_{0}\right) \in$ $X_{T}\left(\bar{G}_{1} \cap \bar{G}_{2}\right) \subseteq X_{T}(\mathbb{C} \backslash F)$. Therefore

$$
\left\langle f_{y_{1}}\left(\lambda_{0}\right)-f_{x_{1}}\left(\lambda_{0}\right), u\right\rangle=0,
$$

that is,

$$
\left\langle f_{y_{1}}\left(\lambda_{0}\right), u\right\rangle=\left\langle f_{x_{1}}\left(\lambda_{0}\right), u\right\rangle .
$$

This shows that $v$ is well defined.

Evidently, $v$ is linear. According to the above argument, there is a constant $M_{b}>0$ such that for every $x \in X$ there are $x_{k} \in X_{T}^{b}\left(\bar{G}_{k}\right), k=1$, 2, satisfying $x=x_{1}+x_{2},\left\|x_{k}\right\|_{b}^{k} \leq M_{b}\|x\|, k=1,2$. So

$$
\begin{aligned}
|\langle x, v\rangle| & =\left|\left\langle f_{x_{1}}\left(\lambda_{0}\right), u\right\rangle\right| \leq\left\|f_{x_{1}}\left(\lambda_{0}\right)\right\|\|u\| \\
& \leq \sup \left\{\left\|f_{x_{1}}(\lambda)\right\| ; \lambda \in \mathbb{C} \backslash \bar{G}_{1}\right\}\|u\| .
\end{aligned}
$$

Thus

$$
|\langle x, v\rangle| \leq\left\|x_{1}\right\|_{b}\|u\| \leq M_{b}\|x\|\|u\| .
$$

This shows that $v$ is bounded, that is, $v \in X^{*}$.

Suppose $x \in X_{T}(\mathbb{C} \backslash F)$. It follows from (2) that there are $x_{k} \in X_{T}^{b}\left(\bar{G}_{k}\right) \subseteq$ $X_{T}\left(\bar{G}_{k}\right), k=1,2$, such that $x=x_{1}+x_{2}$. Thus $x_{1}=x-x_{2} \in X_{T}(\mathbb{C} \backslash F)$. Then there is a closed set $E \subseteq \mathbb{C} \backslash F$ such that $x_{1} \in X_{T}(E)$. Noting $\mathbb{C} \backslash G_{1} \subseteq \mathbb{C} \backslash F$, we may assume without loss of generality that $E \supseteq \mathbb{C} \backslash G_{1}$. Let $g: \mathbb{C} \backslash E \rightarrow X$ be an analytic $X$-valued function such that

$$
(\lambda-T) g(\lambda)=x_{1}, \quad \lambda \in \mathbb{C} \backslash E .
$$

There also exists an analytic $X$-valued function $f_{x_{1}}$ on $\mathbb{C} \backslash \bar{G}_{1}$ such that

$$
(\lambda-T) f_{x_{1}}(\lambda)=x_{1}, \quad \lambda \in \mathbb{C} \backslash \bar{G}_{1} .
$$

Noting that $\mathbb{C} \backslash \bar{G}_{1}$ and $\mathbb{C} \backslash E$ are disjoint, we can define an analytic $X$-valued function on $\left(\mathbb{C} \backslash \bar{G}_{1}\right) \cup(\mathbb{C} \backslash E)=\mathbb{C} \backslash\left(\bar{G}_{1} \cap E\right)$ as

$$
G(\lambda)= \begin{cases}f_{x_{1}}(\lambda), & \lambda \in \mathbb{C} \backslash \bar{G}_{1}, \\ g(\lambda), & \lambda \in \mathbb{C} \backslash E\end{cases}
$$

and it satisfies

$$
(\lambda-T) G(\lambda)=x_{1}, \quad \lambda \in \mathbb{C} \backslash\left(\bar{G}_{1} \cap E\right) .
$$

So $x_{1} \in X_{T}\left(\bar{G}_{1} \cap E\right)$. Then $G\left(\lambda_{0}\right)=f_{x_{1}}\left(\lambda_{0}\right) \in X_{T}\left(\bar{G}_{1} \cap E\right) \subseteq X_{T}(\mathbb{C} \backslash F)$. Hence

$$
\langle x, v\rangle=\left\langle f_{x_{1}}\left(\lambda_{0}\right), u\right\rangle=0 .
$$

This shows $v \in X_{T}(\mathbb{C} \backslash F)^{\perp}$. 
For every $x \in X$ there are $x_{k} \in X_{T}^{b}\left(\bar{G}_{k}\right), k=1,2$, such that $x=x_{1}+x_{2}$, and there exists an analytic $X$-valued function $f_{x_{1}}$ on $\mathbb{C} \backslash \bar{G}_{1}$ such that

$$
(\lambda-T) f_{x_{1}}(\lambda)=x_{1}, \quad \lambda \in \mathbb{C} \backslash \bar{G}_{1} .
$$

Note that $\left(\lambda_{0}-T\right) x_{k} \in X_{T}^{b}\left(\bar{G}_{k}\right), k=1,2$, such that

$$
\left(\lambda_{0}-T\right) x=\left(\lambda_{0}-T\right) x_{1}+\left(\lambda_{0}-T\right) x_{2}
$$

and that $\left(\lambda_{0}-T\right) f_{x_{1}}(\lambda)$ is also an analytic $X$-valued function on $\mathbb{C} \backslash \bar{G}_{1}$ such that

$$
(\lambda-T)\left(\lambda_{0}-T\right) f_{x_{1}}(\lambda)=\left(\lambda_{0}-T\right) x_{1}, \quad \lambda \in \mathbb{C} \backslash \bar{G}_{1} .
$$

So

$$
\begin{aligned}
\left\langle x,\left(\lambda_{0}-T^{*}\right) v\right\rangle & =\left\langle\left(\lambda_{0}-T\right) x, v\right\rangle=\left\langle\left(\lambda_{0}-T\right) f_{x_{1}}\left(\lambda_{0}\right), u\right\rangle \\
& =\left\langle x_{1}, u\right\rangle=\left\langle x_{1}+x_{2}, u\right\rangle=\langle x, u\rangle .
\end{aligned}
$$

Because $x$ is arbitrary, $\left(\lambda_{0}-T^{*}\right) v=u$, that is, $\lambda_{0}-T^{*} \mid X_{T}(\mathbb{C} \backslash F)^{\perp}$ is surjective.

Finally, we prove $X_{T^{*}}^{*}(F) \subseteq X_{T}(\mathbb{C} \backslash F)^{\perp}$. Suppose $u \in X_{T^{*}}^{*}(F)$. Then there exists an analytic $X^{*}$-valued function $f_{u}^{*}: \mathbb{C} \backslash F \rightarrow X^{*}$ such that

$$
\left(\lambda-T^{*}\right) f_{u}^{*}(\lambda)=u, \quad \lambda \in \mathbb{C} \backslash F .
$$

For every $x \in X_{T}(\mathbb{C} \backslash F)$, there is a closed set $E \subseteq \mathbb{C} \backslash F$ such that $x \in X_{T}(E)$; that is, there exists an analytic $X$-valued function $f_{x}: \mathbb{C} \backslash E \rightarrow X$ such that

$$
(\lambda-T) f_{x}(\lambda)=x, \quad \lambda \in \mathbb{C} \backslash E .
$$

We define an analytic complex function on $\mathbb{C}$ as

$$
F(\lambda)= \begin{cases}\left\langle f_{x}(\lambda), u\right\rangle, & \lambda \in \mathbb{C} \backslash E, \\ \left\langle x, f_{u}^{*}(\lambda)\right\rangle, & \lambda \in \mathbb{C} \backslash F .\end{cases}
$$

Note that if $\lambda \in(\mathbb{C} \backslash E) \cap(\mathbb{C} \backslash F)$, then

$$
\begin{aligned}
\left\langle f_{x}(\lambda), u\right\rangle & =\left\langle f_{x}(\lambda),\left(\lambda-T^{*}\right) f_{u}^{*}(\lambda)\right\rangle \\
& =\left\langle(\lambda-T) f_{x}(\lambda), f_{u}^{*}(\lambda)\right\rangle=\left\langle x, f_{u}^{*}(\lambda)\right\rangle ;
\end{aligned}
$$

hence, $F(\lambda)$ is well defined. Because $E \subseteq \mathbb{C} \backslash F$, then $(\mathbb{C} \backslash E) \cup(\mathbb{C} \backslash F)=\mathbb{C}$; hence, $F(\lambda)$ is analytic on the whole plane and

$$
\begin{aligned}
|F(\lambda)| & =\left|\left\langle f_{x}(\lambda), u\right\rangle\right| \leq\left\|f_{x}(\lambda)\right\|\|u\| \\
& =\left\|(\lambda-T)^{-1} x\right\|\|u\| \rightarrow 0
\end{aligned}
$$

as $|\lambda| \rightarrow+\infty$. According to Liouville's theorem, $F(\lambda) \equiv 0$. Hence for $\lambda \in$ $\mathbb{C} \backslash F$,

$$
\left\langle x, f_{u}^{*}(\lambda)\right\rangle=F(\lambda)=0 .
$$

Since $T x \in X_{T}(\mathbb{C} \backslash F)$,

$$
\left\langle T x, f_{u}^{*}(\lambda)\right\rangle=0, \quad \text { for } \lambda \in \mathbb{C} \backslash F .
$$

So for $\lambda \in \mathbb{C} \backslash F$,

$$
\begin{aligned}
\langle x, u\rangle & =\left\langle x,\left(\lambda-T^{*}\right) f_{u}^{*}(\lambda)\right\rangle=\left\langle(\lambda-T) x, f_{u}^{*}(\lambda)\right\rangle \\
& =\lambda\left\langle x, f_{u}^{*}(\lambda)\right\rangle-\left\langle T x, f_{u}^{*}(\lambda)\right\rangle=0,
\end{aligned}
$$

that is, $u \in X_{T}(\mathbb{C} \backslash F)^{\perp}$. The proof is complete. 


\section{REFERENCES}

1. E. Bishop, A duality theorem for an arbitrary operator, Pacific J. Math. 9 (1959), 379-394.

2. N. Dunford and J. Schwartz, Linear operators, Part III, Wiley-Interscience, New York, 1971.

3. C. K. Fong, Decomposability into spectral manifolds and Bishop's property $(\beta)$, Northeast. Math. J. 5 (1989), 391-394.

4. St. Frunză, $A$ duality theorem for decomposable operators, Rev. Roumaine Math. Pures Appl. 16 (1971), 1055-1058.

5. A. L. Shields, Weighted shift operators and analytic function theory, Topics in Operator Theory, Math. Surveys Monographs, vol. 13, Amer. Math. Soc., Providence, RI, 1974.

6. S. L. Sun, Decomposability of weighted shift and hyponormal operators on Hilbert space, Chinese Ann. Math. Ser. A 5 (1984), 575-584.

Department of Mathematics, Jilin University, Changchun, 130023 The People's RePUBLIC OF CHINA 